\title{
Reconfiguration decision making in cognitive wireless network
}

\author{
ZHANG Ping, HE Qian, FENG ZhiYong ${ }^{*} \&$ ZHANG QiXun \\ Wireless Technology Innovation Institute (WTI), Key Laboratory of Universal Wireless Communications of Ministry of Education, Beijing \\ University of Posts and Telecommunications, Beijing 100876, China
}

Received January 18, 2012; accepted April 24, 2012; published online July 12, 2012

\begin{abstract}
Facing the challenges of dynamic adaptation capabilities in the time-varying environment of cognitive wireless networks (CWNs), we introduce reconfiguration capabilities that flexibly and dynamically adapt to changing wireless environments and service requirements. As an essential characteristic of CWNs, the cognitive reconfiguration can meet user requirements, realize interoperability between heterogeneous networks, make full use of radio resources and adapt to time-varying environments to achieve end-to-end requirements. However, the reconfiguration implementation is still challenging due to the need for complex environment cognition, multi-objective optimization, autonomic decision-making and end-to-end requirement extraction. As an intelligent technology for solving complex issues, we apply adaptive neuro-fuzzy inference system (ANFIS) techniques in this paper to address these challenges in cognitive reconfiguration for self-learning and optimal decision making based on multi-domain cognition results. Moreover, this paper designs a generic ANFIS cognitive reconfiguration system including three functional entities, which are the context management module, multi-domain database and ANFIS optimization module. Finally, numerous results prove the effective performance improvements of the ANFIS based reconfiguration solution in CWN for global end-to-end goals.
\end{abstract}

cognitive reconfiguration, cognitive wireless network, adaptive neuro-fuzzy inference system

Citation: Zhang P, He Q, Feng Z Y, et al. Reconfiguration decision making in cognitive wireless network. Chin Sci Bull, 2012, 57: 3713-3722, doi: $10.1007 / \mathrm{s} 11434-012-5255-3$

Cognitive radio (CR) technologies were first proposed for tackling the challenging issues stemming from radio resource limitations because of their capabilities to sense environment, learn historical information and plan intelligently [1]. CR technologies, such as spectrum sensing, dynamic spectrum access and dynamic spectrum sharing mainly focus on improving the spectrum efficiency in a wireless network. With CR technology development in wireless networks, the cognitive wireless network (CWN) concept was proposed as a wireless network composed of cognitive devices and cognitive functions for end-to-end goal enhancement [2]. CWNs manage both the spectrum resource and the network status through the CR capabilities and their own capabilities, such as autonomic computing and self-management [3].

However, recent progress in radio communication not only offers more flexible network services but also makes

\footnotetext{
*Corresponding author (email: fengzy@ bupt.edu.cn)
}

CWNs trends increasingly complex, heterogeneous and dynamic [4]. In this context, CWNs need the capability of enabling their dynamic adaptation in time-varying environments, and moreover, of achieving the end-to-end goals. In this context, reconfiguration is introduced in CWNs as an essential characteristic for meeting user requirements, realizing interoperability between heterogeneous networks, making full use of radio resources and adapting to timevarying environments. In particular, reconfiguration can achieve end-to-end goals and self-management through the reasoning and learning process. The reconfiguration concept has also been studied as a key technology in several research projects, such as the $E^{2} \mathrm{R}$ (end-to-end reconfigurability) project and $E^{3}$ (end-to-end efficiency) projects [5] which aim to design, develop, prototype and showcase solutions that guarantee interoperability, flexibility and scalability between existing legacy and future wireless systems.

Reconfiguration means that both the terminals and the network equipment in a CWN are reconfigurable and can 
intelligently select the best configuration for the environment conditions [3]. In this context, reconfiguration provides the ability to handoff among the heterogeneous networks, optimize the radio configuration and access the appropriate channel, thus meeting the end-to-end goals.

As the next sections discuss, reconfiguration implementation is still challenging because it needs a process of complex environment cognition, multi-objective optimization, autonomic decision-making and end-to-end requirement extraction. In this article, we propose a cognitive reconfiguration architecture to overcome these challenges using adaptive neuro-fuzzy inference system (ANFIS), which has a multi-input and multi-output framework and can deal with complex environmental information and multi-objective optimization. Moreover, the ANFIS can receive the reconfiguration information and end-to-end goals to make decisions automatically through a self-learning mechanism. Finally, the ANFIS is designed as a black box system, which simplifies network operation and saves operating time.

\section{Cognitive reconfiguration}

The initial definition of end-to-end reconfiguration in the $E^{2} R$ white paper [5] states the goal of reconfiguration as follows: "Reconfigurability provides essential mechanisms to terminals and network segments, so as to enable them to adapt to the most appropriate radio access technology (RAT) and also supports the dynamic allocation of resources to RATs". From this definition, the goals which reconfiguration should achieve can be summarized as: (1) Reconfiguration guarantees that users can access the appropriate heterogeneous network flexibly to satisfy application requirements (user requirements); (2) reconfiguration can dynamically allocate the spectrum to avoid transmission congestion in primary user and cognitive user scenarios; (3) reconfiguration can tune configurations of different layers in the protocol stack to improve end-to-end performance in timevarying environments.

However, in order to achieve the reconfiguration goals in CWN, cognitive abilities should be applied in reconfiguration process. The main challenges of cognitive enabled reconfiguration to achieve these goals are summarized by cognition, interpretability, complexity and autonomy, which will be discussed in detail below.

\subsection{Challenges of cognitive reconfiguration}

(i) Cognition. To make the best reconfiguration decision for meeting the end-to-end goal, the CWN is required to be able to span all configurations from different protocol layers, the available resource (including available channels and interface etc.) in heterogeneous networks and also environmental information. However, the methods which can col- lect parameters from different domains are specific. For instance, technologies for sensing the configuration from different layers (physical, medium access, network, transport, and application) are diverse. Moreover, with the increasing number of parameters involved in awareness process, a lot of time is wasted in detection and the computational load is also increased. In fact, the information sharing mechanism in a CWN relieves some of the cognitive load. Users in the CWN share the gathered information and previous experience with each other which makes cognition effective and decision-making faster. However, if there are many parameters with different properties, cognition will remain a conundrum.

(ii) Interpretability. As stated above, a CWN needs to select a knowledge database and a transmission approach in the design to achieve information exchange among the users. The challenge related to information interpretability is that the information stored in the database must be understandable by all the users. Moreover, to maintain data coherence, the information should also be retained correctly in the transmission process. Context information should be transmitted to user terminals and network nodes by suitable enablers, such as a cognitive pilot channel (CPC), which can improve the quality of the information transmission. However, the introduction of CPC resolves the challenges as it is defined to carry several types of information and maximizes the reactivity of network entities to any context change in a dynamic scenario [6].

(iii) Complexity. The end-to-end goal of reconfiguration takes the system requirements and performance with an end-to-end view. The end-to-end performance is determined by combining the performance of each protocol layer. Since the performance of each layer is determined by the parameters for that layer, the performance computation becomes more complex as the number of parameters in the reconfiguration increases. In other words, extracting the end-to-end goal is related to the number of complex multi-domain configuration parameters and performance parameters, and is a complex computational process.

(iv) Autonomy. The autonomic and self-x concepts should be introduced in the reconfiguration for adapting to the dynamic environment. Since the dynamic environment varies without regulation, artificial reconfiguration decision-making needs more time and offers a delayed decision without intelligence. In this situation, autonomic organization, reasoning, learning and decision-making are essential. Depending on the context, "self-x" can actually refer to aspects such as configuration, planning, optimization, healing, re-tuning, and resource re-allocation, which may be applied to the edges of the network and/or the user terminals [5]. Solving this challenge could lead to an increased degree of automation in the operation of CWNs. Autonomy can be seen as a solution to efficient management of cognitive heterogeneous wireless systems. 


\subsection{Related work}

The majority of existing reconfiguration research is oriented toward several definite fields. Actually, the $\mathrm{E}^{2} \mathrm{R}$ project has made progress in reconfiguration architectures such as reconfiguration controller [5], reconfiguration business system architecture [5] and reconfiguration management architecture $[7,8]$. The reconfiguration controller is used to control transmission and configuration. Reconfiguration business system architecture focuses on cooperation and competition between operators in different scenarios. The reconfiguration management architecture achieves integrated management through multiple function entities.

Many recent proposals rely on multifarious technologies, such as design of experiment (DOE) [9-11], and seem to be well suited to handling large sets of multi-domain variables and carrying out reconfiguration decision for selecting the configuration meeting specified design objectives. However, a major drawback of this technique is that it is too computationally intensive. That is to say, the computational process preceding decision making may be unbearable.

Many recent publications propose the use of artificial intelligence techniques for reconfiguration in CWNs. Ant colony optimization (ACO) has been proposed for routing reconfiguration [12] and parameter reconfiguration [13], and, more recently, resource reconfiguration [14,15] (such as channel reallocation, spectrum occupancy and so on). These problem-specific solutions, however, are not reusable for a general-purpose cross-layer optimization engine. Swarm intelligence (artificial fish school algorithm) can help to reconfigure resource allocation [16]. Artificial neural networks (ANNs) provide a general, practical method for learning either real-valued or discrete-valued functions from examples $[17,18]$. It offers interesting learning capabilities in reconfiguration decision-making. However, the computational process for ANNs cannot take imprecision and uncertainty issues into account, as required by a generic reconfiguration system.

Finally, a few works [19,20] use ANFIS for transmission rate prediction and network handoff decision-making. These proposals, however, are targeted at specific scenarios: channel stats estimation in cognitive radio system [19] and vertical handoff in wireless heterogeneous networks [20]. No effort is made to generalize ANFIS to different cognitive reconfiguration problems. To the best of our knowledge, our approach is novel, as no previous research uses ANFIS as a generic model for reconfiguration in CWNs, which can deal with complex environmental information and multi-objective optimization problems with the self-learning ability.

\section{ANFIS}

ANFIS is designed as a rule-based predictor incorporating rule-based reasoning and learning. ANFIS is a combination of two functional processes: fuzzy converting and ANN computation. In this context, ANFIS possesses the advantages of both of them, and moreover, removes the disadvantages by interaction of two processes. Fuzzy reasoning combining ANN computation can adaptively adjust membership functions, cancel wrong rules, update new rules and decrease the dependence of existing rules. ANN computation with fuzzy logic can decrease the learning duration as compared with individual ANN computations.

\subsection{Fuzzy reasoning}

(i) Fuzzy set. Fuzzy set theory differs from traditional set theory in that partial membership is allowed [2]. A fuzzy set can be defined as a set with a fuzzy boundary with membership to indicate the extent to which an element belongs to the set.

(ii) Membership. In fuzzy-based conversion, the input to the CWN is converted to a fuzzy description. This conversion relies on calculating the degree of membership, which is represented by a real value in $[0,1]$ where 0 and 1 correspond to full non-membership and full membership respectively [2].

(iii) Membership function. The membership function can be written as:

$$
\mu_{A}(x): X \rightarrow[0,1] .
$$

For each $x \in X$, the degree of membership in $A$ is given by $\mu_{A}(x)$. In general, triangular or trapezoidal functions are used as membership functions because of their simplicity.

\subsection{ANN-based computation}

As illustrated in Figure 1, the ANFIS model has a five-layer structure.

(i) Input layer. The input layer is the first layer in the ANFIS model and receives the values of the radio parameters. Every neuron stands for a specific input, so the parameters are classified and sent to the corresponding neuron in this layer.

(ii) Input fuzzification layer. The input fuzzification layer stores all the membership functions for the radio parameters. The input parameters are converted to degree of membership using these functions.

(iii) Fuzzy rule layer. Every neuron in the fuzzy rule layer corresponds to a fuzzy rule. The output of this layer is the product of all the inputs:

$$
\mu_{R_{i}}=\mu_{A_{1}} \times \ldots \times \mu_{A_{i}},
$$

where $\mu_{A_{i}}$ is the degree of membership from the input fuzzification layer and $\mu_{R_{t}}$ is the intensity of activation of the $i$ rule. 


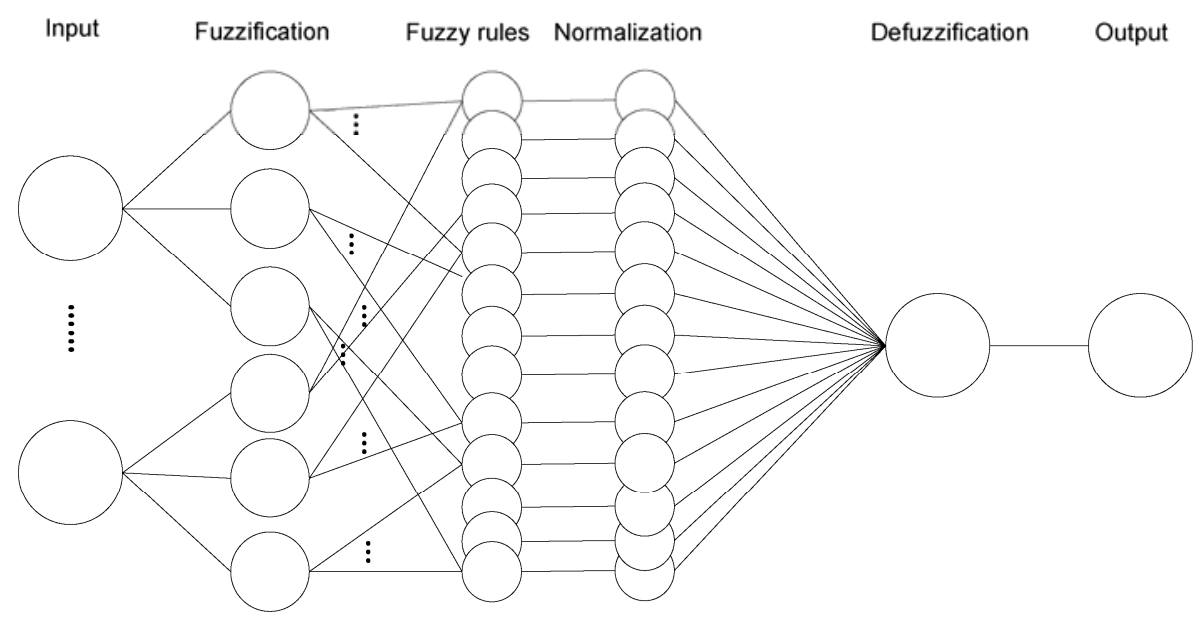

Figure 1 Structure of the ANFIS model.

(iv) Normalization layer. The number of neurons in this layer is the same as that in the fuzzy rule layer. Every normalization neuron connects to a neuron in the fuzzy rule layer to normalize the result in $[0,1]$. Normalization represents the level of influence level of each rule in decision making, and is calculated as:

$$
\overline{\mu_{R_{i}}}=\mu_{R_{i}} / \sum_{j=1}^{n} \mu_{R_{j}},
$$

where $\sum \mu_{j}$ is the sum of all the degree of membership from the fuzzy rule layer.

(v) Defuzzification layer. The neuron in this layer receives the normalization results and the initial input $\left(x_{1}, \ldots, x_{m}\right)$ and defuzzifies as follows:

$$
y_{i}=\overline{\mu_{R_{i}}} \times\left(k_{i 0}+k_{i 1} x_{1}+\ldots+k_{i m} x_{m}\right),
$$

where $k_{\text {in }}$ is the adjustment parameter of the rule conclusion. These defuzzification results are merged as follows:

$$
\begin{aligned}
y & =\sum_{i=1}^{n} \overline{\mu_{R_{i}}} \times\left(k_{i 0}+k_{i 1} x_{1}+\ldots+k_{i m} x_{m}\right) \\
& =A k
\end{aligned}
$$

$$
\begin{aligned}
& \text { s.t. }
\end{aligned}
$$

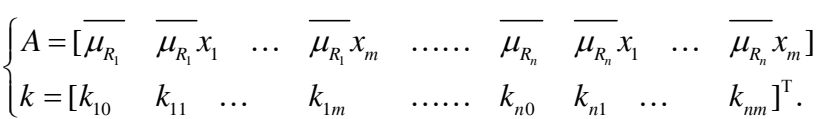

Compared with the desired output $y_{d}$, the deviation is $y_{d}-A k$. Using least-square estimation to minimize the deviation, the estimate for $k$ is:

$$
k=\left(A^{\mathrm{T}} A\right)^{-1} A^{\mathrm{T}} y_{d} .
$$

\subsection{ANFIS decision-making}

An ANFIS decision-making system can determine a decision using fuzzy reasoning and ANN learning. Since the system takes into account imprecision and uncertainty issues, the fuzzification and defuzzification processes are essential to translate to and from the fuzzy representation. The ANFIS decision-making framework, which includes the key components and their relationships, is illustrated in Figure 2. In this framework, the reasoning and learning engine are the primary functional entities. Moreover, the system has two kinds of mechanism for data storage to facilitate reasoning and learning: the rule engine manages the rules for reasoning and the knowledge database stores the experience and real-time environment information for learning. To embody initiative in the system and save computation time, the reasoning engine builds a priori analysis to reach a decision. The learning engine optimizes the result of the reasoning engine through computation using information in the knowledge database, and then stores the result in the database for future reference. The decision is derived from the result of reasoning and learning.

\section{ANFIS cognitive reconfiguration}

In this section, we prove that the advantages of ANFIS allow it surmount the challenges of cognitive reconfiguration. First, the fuzzy rules used in ANFIS cognitive reconfiguration simplify the cognition process through abstraction. In this context, the rules use fuzzy logic to reduce information storage. Second, the multi-domain cognitive database introduced in the ANFIS reconfiguration framework implements information interpretability for the information storage and classification mechanism. Third, the ANFIS reconfiguration system has multi-objective optimization ability and can analyze end-to-end goals and react to complex situations. Finally, the prediction ability of ANFIS systems can help user terminals and network nodes to make reconfiguration decisions ahead of time, thus addressing the autonomy challenge. 


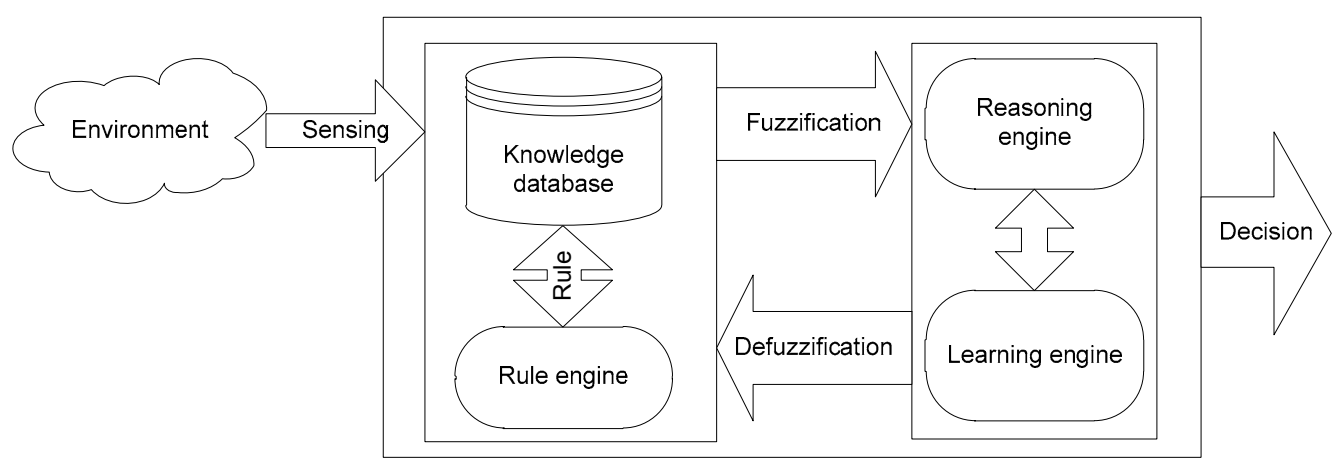

Figure 2 The reconfiguration decision-making framework.

\subsection{ANFIS cognitive reconfiguration architecture}

To achieve cognitive reconfiguration in CWNs, we define the following principles for the ANFIS cognitive reconfiguration model:

(1) The reconfiguration decision-making process is carried in the cognitive engine.

(2) The terminals in the CWN are intelligent and change the configuration dynamically.
(3) The reconfiguration decision-making is complete with both the user terminals and network nodes.

To present these principles in our proposed system, we need to introduce critical functional entities in both the user terminals and the network nodes. In this context, the generic architecture of cognitive reconfiguration decision-making is shown in Figure 3.

The user terminals include the context manager, ANFIS

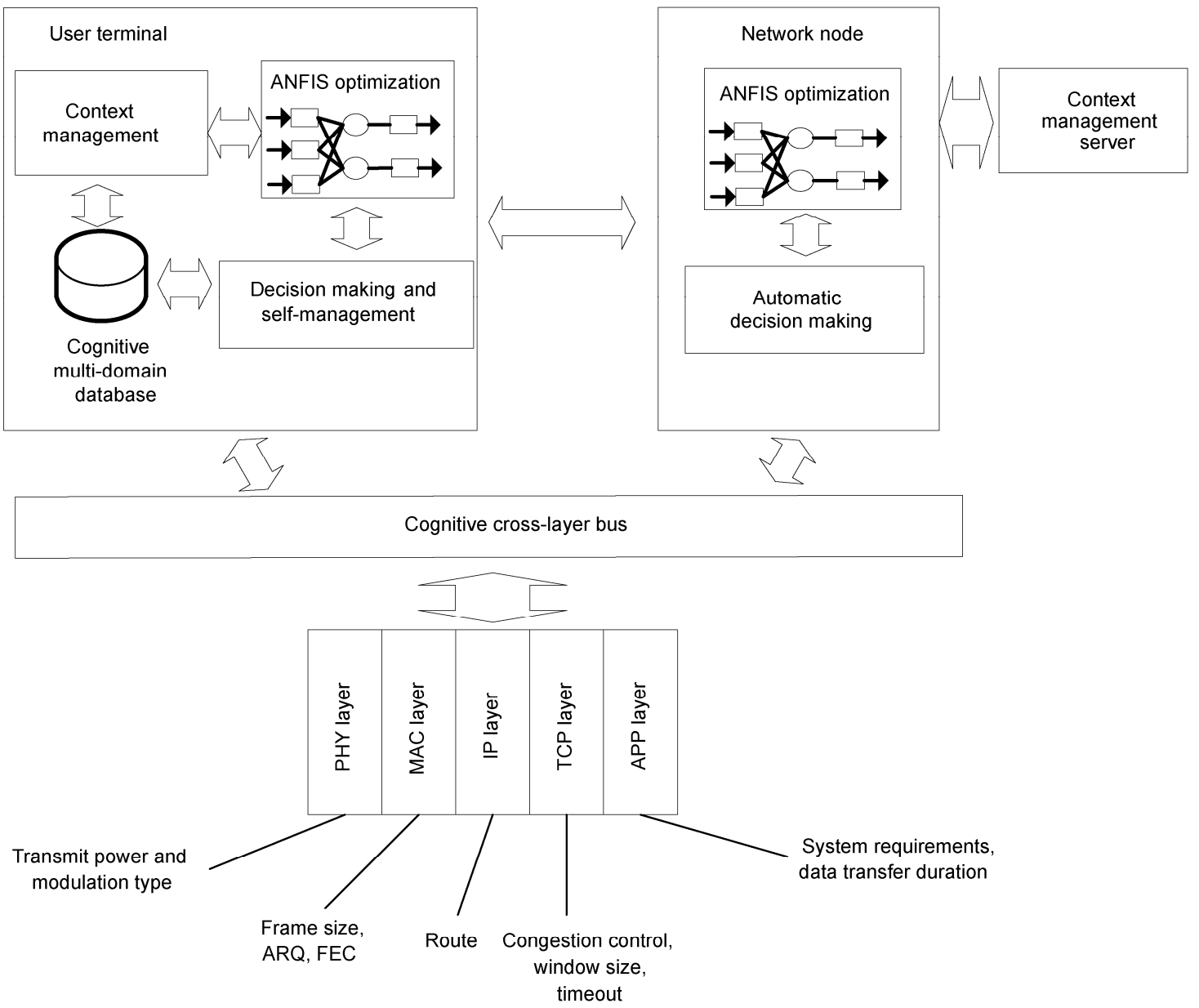

Figure 3 Generic ANFIS cognitive reconfiguration architecture. 
optimization entity and self-management entity. The network nodes include the context management server and automatic decision-making entity as well as the ANFIS optimization entity. The context management server handles requests for reconfiguration and obtains available information. The parameters from different layers transmit to both user terminals and network nodes for decision-making through the cognitive cross-layer bus.

\subsection{Fuzzy rules in ANFIS reconfiguration systems}

In CWNs, predicting network performance with existing knowledge is indispensable for avoiding performance deterioration and for decision-making in reconfiguration. We realize reasoning based on the fuzzy rules stored in the database as "IF $s_{i}$ is $A$ THEN $H_{j}$ is $C$ " format, which influences the system performance because of the changing radio parameters. The variables $s_{i}(i \in(1, n))$ are the radio parameters, such as channel coding, transmission power, location, modulation schemes, channel bandwidth, type of service and so on. $H_{j}(j \in(1, m))$ are the system performance parameters, such as system throughout, reliability and so on. $A$ and $C$ are the linguistic attributes of the parameters and may be low, medium or high.

Therefore, fuzzy reasoning predicts system performance from distinct parameters using the fuzzy rules. The reason is that complex information in the CWN can be more easily generalized with fuzzy rules than with definite rules. Moreover, definite rules occupy more storage space than a fuzzy rule as a fuzzy statement can substitute for several definite rules. Fuzzy rules simplify the cognition process to some extent. Fuzzy rule-based reasoning in the ANFIS reconfiguration system is quicker and considers imprecision and uncertainty issues which is required in a generic reconfiguration system.

\subsection{Multi-domain database}

To implement information interpretability in an ANFIS reconfiguration system, sensing results will be stored in a multi-domain cognitive database. Data in the multi-domain cognitive database are divided into five domains: the radio domain, the network domain, the user domain, the policy domain and the rule domain. Users have a common view of different information in a specific domain: parameters related to radio transmission characteristics are stored in the radio domain, parameters related to network status are in the network domain, information related to user requirements is in the user domain, and policies related to radio resource management are in the policy domain. In particular, the rule domain stores fuzzy rules for fuzzy reasoning in the ANFIS reconfiguration system. The multi-domain cognitive database can achieve interpretability and also make information extraction faster.

\subsection{Multi-objective optimization based ANFIS}

The ANFIS is a multi-input and multi-output system that can overcome the complexity challenge. Configurations input into the ANFIS model allow performance results to be deduced from the model after the computational process. It is worth mentioning that the computational process is carried out by the ANFIS internal mechanism, and thus avoids the network and users from bearing the computational load.

The characteristics of ANFIS make it appropriate for computing the influence of complex multi-domain data on the end-to-end goal, which is significant for cognitive reconfiguration decision-making. The multi-objective optimization process is presented in the ANFIS model to demonstrate that the ANFIS reconfiguration system has the capability to analyze complex multi-domain parameters and extract the end-to-end goal.

(i) Step 1: Initializing fuzzy inference system.

Initialize the membership functions of radio parameters. Specifically, define the abscissa value of inflection points in the function curves as a $p \times q$ matrix $x^{\prime}$ ( $p$ is the number of parameters and $q$ is the number of fuzzy sets). Load the training data and calculate the number of rules:

$$
n=a C(b, 1) C(c, 1)+b C(a, 1) C(c, 1)+c C(a, 1) C(b, 1),
$$

where $a, b, c$ are the membership function numbers of the radio parameters, and $C($, denotes the combination number.

(ii) Step 2: Training and optimizing process.

Set the cycle index as $m=1$.

In the training process, end-to-end indicators can be deduced from the training data and $x^{\prime}(m)$ through the operations of the input layer, fuzzification layer, fuzzy rules layer, normalization layer, and defuzzification layer. In the optimization process, $x^{\prime}$ is then regulated adaptively as the cycle index increases. This adjustment factor is the error matrix $\Delta x^{\prime}(m)$, which is calculated from eq. (5) and the concatenation rule defined in [21].

$$
\begin{aligned}
& \Delta x_{p q}^{\prime}(m)=-\alpha \frac{\partial E}{\partial x_{p q}^{\prime}(m)} \\
& =-\alpha \frac{\partial E}{\partial e} \times \frac{\partial e}{\partial y} \times \frac{\partial y}{\partial y_{i}} \times \frac{\partial y_{i}}{\partial \overline{\mu_{R_{i}}}} \times \frac{\partial \overline{\mu_{R_{i}}}}{\partial \mu_{R_{i}}} \times \frac{\partial \mu_{R_{i}}}{\partial \mu_{p q}} \times \frac{\partial \mu_{p q}}{\partial x_{p q}^{\prime}(m)} \\
& =\alpha e\left(k_{i 0}+k_{i 1} x_{1}+k_{i 2} x_{2}+k_{i 3} x_{3}\right) \frac{\overline{\mu_{R_{i}}}\left(1-\overline{\mu_{R_{i}}}\right)}{\mu_{R_{i}}} \times \frac{\mu_{R_{i}}}{\mu_{p q}} \times \frac{\partial \mu_{p q}}{\partial x_{p q}^{\prime}(m)} \\
& =\alpha e\left(k_{i 0}+k_{i 1} x_{1}+k_{i 2} x_{2}+k_{i 3} x_{3}\right) \times \frac{\overline{\mu_{R_{i}}}\left(1-\overline{\mu_{R_{i}}}\right)}{\mu_{p q}} \times \frac{\partial \mu_{p q}}{\partial x_{p q}^{\prime}(m)} \\
& \text { s.t. }\left\{\begin{array}{l}
e=y_{d}-y, \\
E=0.5\left(y_{d}-y\right)^{2},
\end{array}\right.
\end{aligned}
$$

where $\alpha$ is the learning speed, $E$ is the instantaneous square-error, and $y$ and $y_{d}$ are the actual output and desired output of the ANFIS model. 
After updating $x^{\prime}(m)$, the cycle index becomes $m=m+1$ and returns to the beginning of the loop.

(iii) Step 3: Reconfiguration decision making.

After the training process, the ANFIS model learns the relationship between complex multi-domain parameters and end-to-end indicators. That is, the trained ANFIS system deduces the corresponding end-to-end performance when new parameters are input into the system. The ANFIS cognitive reconfiguration system will make a reconfiguration decision to meet end-to-end goals after comparing the performance indicators with the network goals.

\subsection{Autonomic decision-making based ANFIS}

While collaborative decision-making requires the interaction between network nodes and user terminals, any autonomous approach should introduce advantages in decisions-making. In this context, two target areas are identified: (1) the network nodes autonomously adapt to the timevarying needs of the system from a network perspective; and (2) the user terminals exchange context information, system requirements and reconfiguration decisions with neighboring entities and network nodes.

The ANFIS cognitive reconfiguration system is located in both the user terminals and network nodes. The ANFIS system can adjust membership functions and rules using the training data to guarantee the validity and optimality of reasoning. The trained ANFIS cognitive reconfiguration
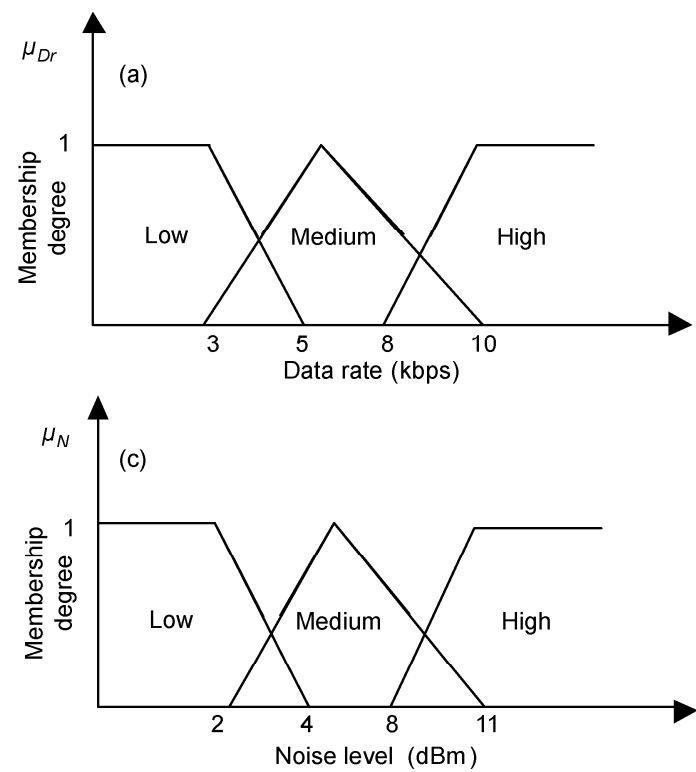

system then has the capability to predict the network state, helping the network to know the alteration of state ahead of time and automatically prepare for reconfiguration decision-making.

\section{Proof of concept}

The data rate, transmission power and noise level are chosen as the radio environment parameters, as they are in different protocol layers and embody complexity of cognition in simulations. Moreover, they are the primary factors that affect performance in general communication systems, while noise level is the ordinary environmental factor that affects wireless communication. To realize a reconfiguration process that is able to adjust the cross-layer parameters to meet the specified performance goals in this simulation, we denote the throughput (representing system performance) metric by $T$. The subscripts $D r, N$ and $T p$ respectively denote data rate, noise level, and transmission power (representing the radio environment parameters). The initial membership functions are defined as in Figure 4, where the threshold values correspond to previously gathered data [9]. These parameters are chosen to represent their effects on throughput in three situations: the setting optimized by the ANFIS algorithm, the best case setting and the worst setting. The configurations shown in Table 1 are the radio performances that produce the best and worst performance.
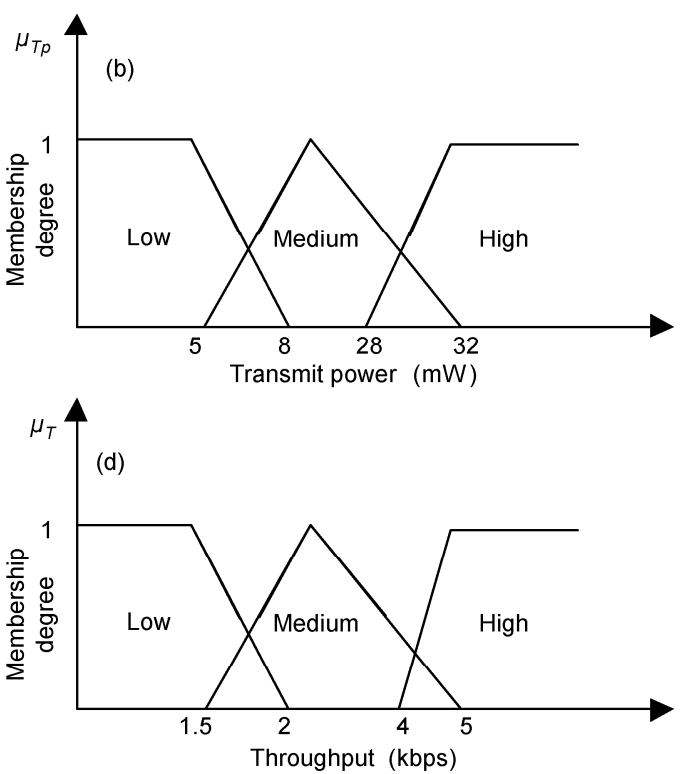

Figure 4 Fuzzy membership function curves. (a) Data rate membership function; (b) transmit power membership function; (c) noise membership function; (d) throughput membership function.

Table 1 Best and worst case static configuration

\begin{tabular}{cccc}
\hline & Data rate $(\mathrm{kbps})$ & Transmit power $(\mathrm{mW})$ & Noise $(\mathrm{dBm})$ \\
\hline Best & 11 & 50 & 5 \\
Worst & 1 & 8 & 10.3 \\
\hline
\end{tabular}


However, Figure 4 only presents the membership functions based on gathered data and thus cannot represent the current radio environment. In this context, we improve these membership functions through inputting real-time performance and radio data to the ANFIS system to obtain realtime membership as shown in Figure 5. Figure 5 shows the trained membership function curves for data rate, transmission power and noise level through training in the ANFIS system. Compared with Figure 4, these membership functions approximate the actual situation as they adjust according to the training data. It is worth mentioning that the trained membership allows more accurate reconfiguration decision-making.

Meanwhile, the ANFIS system generates the rules that describe the effect of the radio parameters on throughput by analyzing the real-time data. Figure 6 shows the effect obtained by analysis during the training process: (a), (b), (c) show the variation trend curves for throughput when a single radio parameter changes; (d), (e), (f) show the throughput variations when two radio parameters change simultaneously. Based on these influences between radio parameters and throughput, a reconfiguration decision is made to generate a series of configurations able to meet the specified performance goals.

After the training and adaptation process, the generated model allows adaptation of the radio configuration in response to changing environmental conditions or goals. The neuro-fuzzy reconfiguration algorithm starts with the most conservative setting, equivalent to the worst setting. It then adapts to meet performance goals using the trained ANFIS model. We compare the performances of the best and worst case static configurations to the ANFIS reconfiguration algorithm with respect to throughput when changing the radio parameters. The scenario is as follows. The duration of the simulation is $180 \mathrm{~s}$. Noise is transmitting at $0.81 \mathrm{dBm}$ from
40 to $70 \mathrm{~s}$, and at $10.3 \mathrm{dBm}$ from 70 to $160 \mathrm{~s}$. The data rate is increased to $10 \mathrm{kbps}$ from 20 to $90 \mathrm{~s}$ and dropped back to $3 \mathrm{kbps}$ from 130 to $180 \mathrm{~s}$. The transmission power is 25 $\mathrm{mW}$ from 60 to $90 \mathrm{~s}$ and is adjusted to $11 \mathrm{~mW}$ from 140 to $180 \mathrm{~s}$. Therefore, the radio must contend with changes in the noise environment as well as in system load. Figure 7 shows the best and worst static configurations compared with the optimized configurations based on the neuro-fuzzy reconfiguration algorithm with respect to throughput. Therefore, the results denote that the reconfiguration algorithm is able to adjust radio parameters and elevate the system performance so that it meets the system goals. As depicted in Figure 7 , reconfiguration significantly increases throughput relative to the static configurations.

Figure 8 demonstrates changes in the radio parameters for three situations of defined dynamic environment. It can be seen that the static configurations are entirely under the control of the environment and adopt no measures. Meanwhile, reconfiguration adjusts (increases or decreases) the parameters according to the effect of the dynamic environment on network performance. Moreover, it is worth mentioning that the reconfiguration algorithm will not carry out endless optimization. Instead, it terminates tuning when the system goal is satisfied, such as when the data rate remains unchanged from 160 to $180 \mathrm{~s}$.

\section{Conclusion}

This article proposes a novel reconfiguration decisionmaking approach, based on an improved decision framework, to solve the challenges of cognitive reconfiguration implementation. Different aspects of these challenges are presented and discussed, such as complex environment cognition, multi-objective optimization, autonomic decision-
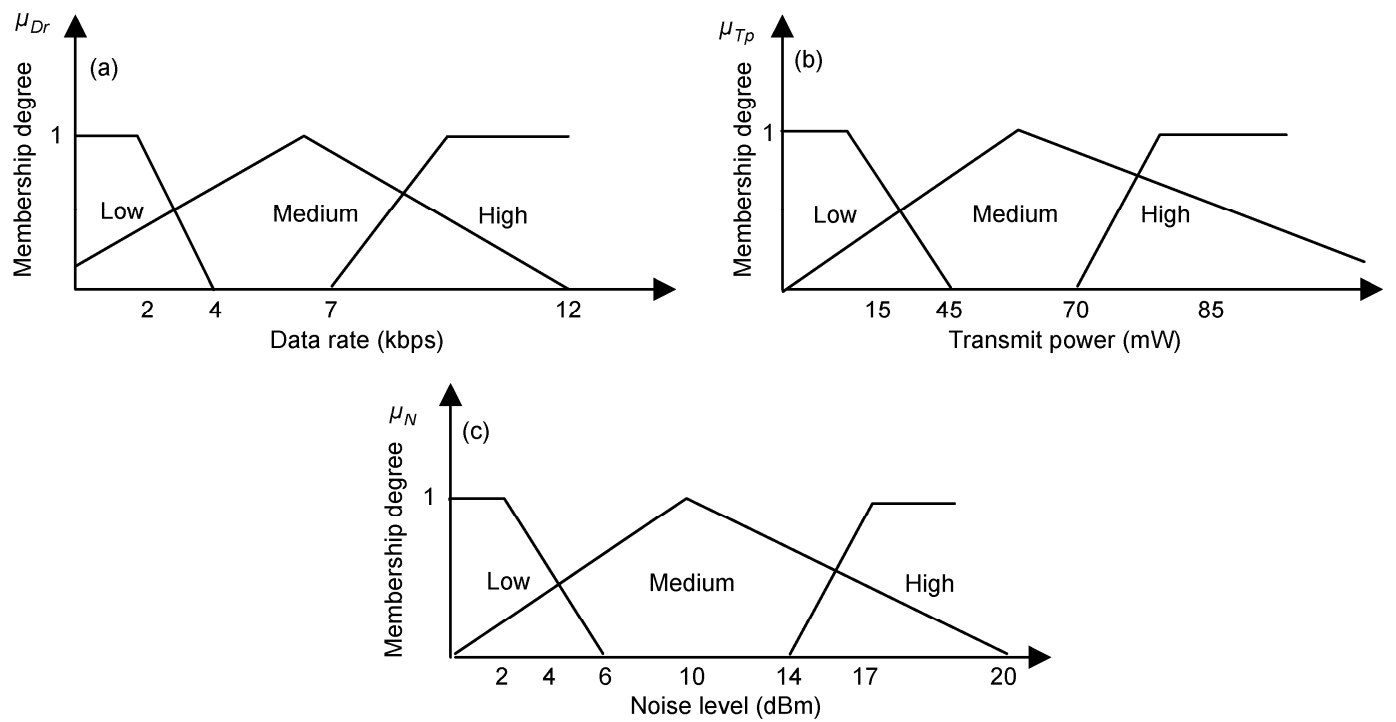

Figure 5 Training results - trained membership function curves. (a) Trained data rate membership function; (b) trained membership transmit power function; (c) trained noise membership function. 

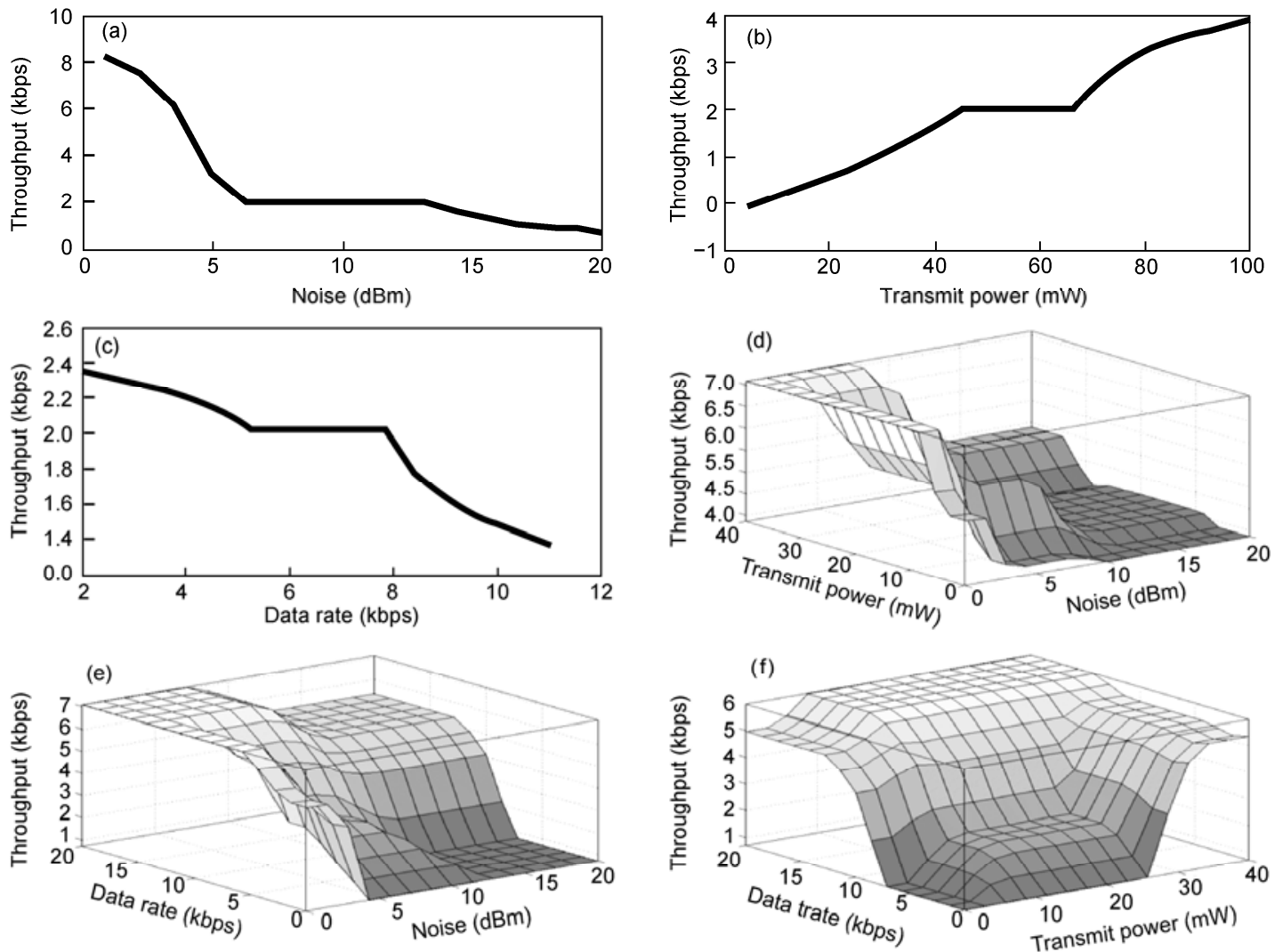

Figure 6 (Color online) Effect of radio parameters on system performance. (a) Effect of noise level on throughput; (b) effect of transmit power on throughput; (c) effect of data rate on throughput; (d) effect of noise level and transmit power on throughput; (e) effect of noise level and data rate on throughput; (f) effect of data rate and transmit power on throughput.

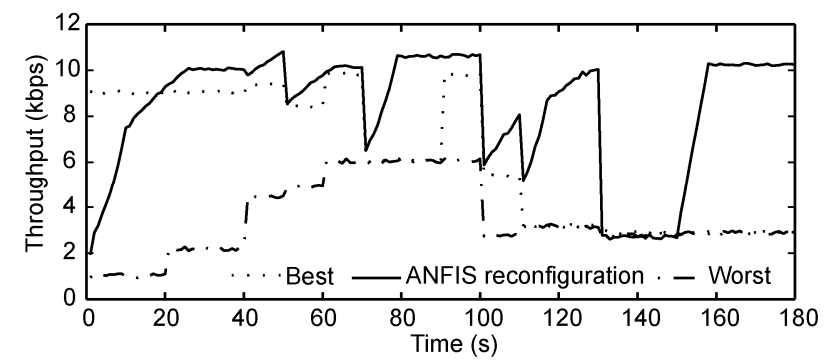

Figure 7 ANFIS reconfiguration algorithm compares with best and worst static configuration on throughput.

making and end-to-end requirement extraction. Then the ANFIS approach is applied to reconfiguration decisionmaking to overcome these challenges. The fuzzy rules used in the ANFIS cognitive reconfiguration simplify the cognition process through abstraction, the multi-objective optimization ability of ANFIS reconfiguration systems analyze the end-to-end goals and react to complex situations, and the prediction ability of ANFIS systems help the user terminals and network nodes to make reconfiguration decisions ahead of time. Furthermore, results show that ANFIS reconfiguration can improve the system performance.
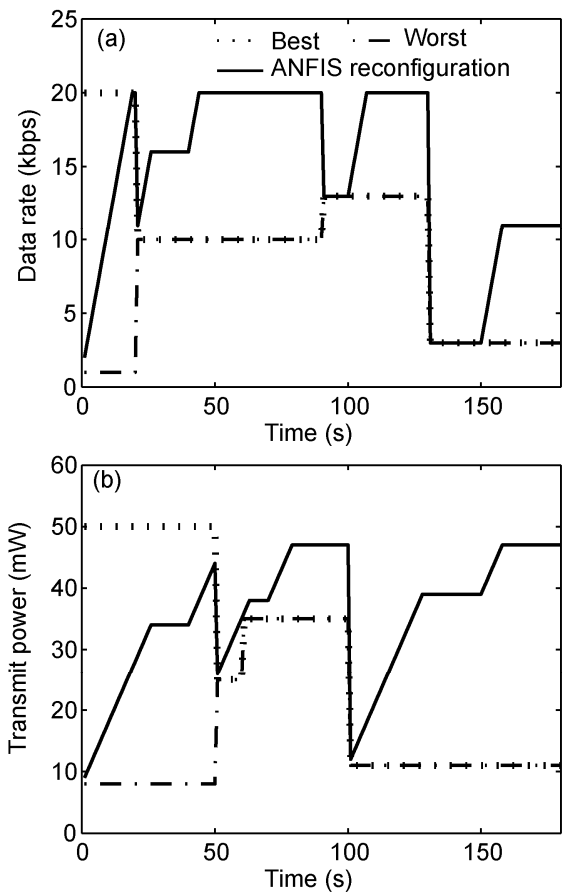

Figure 8 The alteration of data rate and transmission power with changing noise levels in three situations. (a) The data rate curve in three situations; (b) the transmit power curve in three situations. 
This work was supported by the National Basic Research Program of China (2009CB320400), the National Natural Science Foundation of China (60832009, 61121001), the National Science and Technology Major Project of China (2012ZX03003006).

1 Mitola J, Maquire J, Gerald Q. Cognitive radio: Making software radios more personal. IEEE Pers Commun, 1999, 6: 13-18

2 Nicola B, Michele Z. Fuzzy logic for cross-layer optimization in cognitive radio networks. IEEE Commun Mag, 2008, 46: 64-71

3 Demestichas P, Dimitrakopoulos G, Strassner J, et al. Introducing reconfigurability and cognitive networks concepts in the wireless world. IEEE Veh Technol Mag, 2006, 1: 32-39

4 Thomas R W, Friend D H, Dasilva L A, et al. Cognitive networks: Adaptation and learning to achieve end-to-end performance objectives. IEEE Commun Mag, 2006, 44: 51-57

5 George D, Flora M, Karim E K, et al. Scenarios, System Requirements and Roadmaps for Reconfigurability. Technical Report. IST Wireless World Research Forum, 2004

6 Feng Z Y, Zhang Q X, Tian F, et al. Novel research on cognitive pilot channel in cognitive wireless network. Wireless Pers Commun, 2012, 62: $455-478$

7 Muck M, Bourse D, Moessner K, et al. End to end reconfiguability in heterogeneous wireless systems-software and cognitive radio solutions enriched by policy- and context-based decision making. In: Proceedings of the 16th IST Mobile and Wireless Communications Summit, 2007 Jul 1-5, Budapest, Hungary. Washington DC: IEEE, 2007. 1-5

8 Boufidis Z, Alonistioti N, Holland O, et al. End-to-end architecture for cognitive reconfigurable wireless networks. In: Proceedings of the 16th IST Mobile and Wireless Communications Summit, $2007 \mathrm{Jul}$ 1-5, Budapest, Hungary. Washington DC: IEEE, 2007. 1-5

9 Weingart T, Sicker D C, Grunwald D. A statistical method for reconfiguration of cognitive radios. IEEE Wirel Commun, 2007, 14: 34-40

10 Weingart T, Sicker D C, Grunwald D. A method for dynamic configuration of a cognitive radio. In: Proceedings of the 1st IEEE Workshop on Networking Technologies for Software Defined Radio Networks, 2006 Sept 25, Reston, VA, USA. Washington DC: IEEE, 2006. 93-100

11 Weingart T, Gary V Y, Douglas C, et al. Implementation of a reconfiguration algorithm for cognitive radio. In: Proceedings of the 2nd Cognitive Radio Oriented Wireless Networks and Communications,
2007 Aug 1-3, Orlando, FL, USA. Washington DC: IEEE, 2007. $171-180$

12 Song Z, Shen B, Zhou Z, et al. Improved ant routing algorithm in cognitive radio networks. In: Proceedings of the 9th International Symposium on Communications and Information Technology, 2006 Sept 28-30, Icheon, Korea. Washington DC: IEEE, 2009. 110-114

13 Zhao N, Li S, Wu Z. Cognitive radio engine design based on ant colony optimization. Wireless Pers Commun, 2011, 2011: 1-10

14 Andreotti R, Stupia I, Giannetti F, et al. Resource allocation in OFDMA underlay cognitive radio systems based on ant colony optimization. In: Proceedings of the IEEE Eleventh International Workshop on Signal Processing Advances in Wireless Communications, 2010 Jun 20-23, Marrakech, Morocco. Washington DC: IEEE, 2010. 1-5

15 Huang W, Chen J, Li S. A channel allocation algorithm for minimizing handoff rate in cognitive radio networks. In: Proceedings of the 4th International Conference on Wireless Communications, Networking and Mobile Computing, 2008 Oct 12-14, Dalian, China. Washington DC: IEEE, 2008. 1-4

16 Doerr C, Sicker D C, Grunwald D. Dynamic control channel assignment in cognitive radio networks using swarm intelligence. In: Proceedings of the 2008 IEEE Global Telecommunications Conference, 2008 Nov 30-Dec 4, New Orleans, LA, USA. Washington DC: IEEE, 2008. 1-6

17 Baldo N, Zorzi M. Learning and adaptation in cognitive radios using neural networks. In: Proceedings of the 5th IEEE Consumer Communications and Networking Conference, 2008 Jan 10-12, Las Vegas, NV, USA. Washington DC: IEEE, 2008. 998-1003

18 Adamopoulou E, Demestichas K, Theologou M. Enhanced estimation of configuration capabilities in cognitive radio. IEEE Commun Mag, 2008, 46: 56-63

19 Çalhan A, Çeken C. An adaptive neuro-fuzzy based vertical handoff decision algorithm for wireless heterogeneous networks. In: Proceedings of the IEEE 21st International Symposium on Personal Indoor and Mobile Radio Communications, 2010 Sept 26-30, Istanbul, Turkey. Washington DC: IEEE, 2010. 2271-2276

20 Hiremath S, Patra S K. Transmission rate prediction for cognitive radio using adaptive neural fuzzy inference system. In: Proceedings of the International Conference on Industrial and Information Systems, 2010 Jul 29-Aug. 1, Karnataka, India. Washington DC: IEEE, 2010. 92-97

21 Michael N. Artificial Intelligence: A Guide to Intelligent Systems. 2nd ed. London: Pearson Education, 2005

Open Access This article is distributed under the terms of the Creative Commons Attribution License which permits any use, distribution, and reproduction in any medium, provided the original author(s) and source are credited. 